\title{
A Parametric Study of Mental Spatial Transformations of Bodies
}

\author{
J effrey M. Zacks,* J ohn M. Ollinger,* Margaret A. Sheridan,* and Barbara Tversky† \\ *Department of Psychology, Washington University, St. Louis, Missouri 63130-4899; and †Stanford University.
}

Received August 2, 2001

\begin{abstract}
Two classes of mental spatial transformation can be distinguished: Object-based spatial transformations are imagined movements of objects; and egocentric perspective transformations are imagined movements of one's point of view. The hypothesis that multiple neural systems contribute to these mental imagery operations was tested with functional MRI. Participants made spatial judgments about pictures of human bodies, and brain activity was analyzed as a function of the judgment required and the time taken to respond. Areas in right temporal, occipital and parietal cortex and the medial superior cerebellum appear to be differentially involved in object-based spatial transformations. Additionally, midline structures and lateral parietal cortex were found to decrease in activity during the spatial reasoning tasks, independently of the judgment required or of the latency of response. The results are discussed in terms of a model of spatial reasoning that postulates specialized subsystems for performing object-based and egocentric perspective image transformations. 2002 Elsevier Science (USA)
\end{abstract}

\section{INTRODUCTION}

Whenever a surgeon conducts endoscopic surgery, or a chemist builds a model for a protein, or a friend contemplates how to arrange furniture in a new home, they are engaging in feats of complex spatial reasoning. Components of spatial reasoning can be selectively impaired following brain injury, which indicates a certain degree of functional localization. This in turn suggests that an effective way to understand how the brain performs such tasks is to identify the neural systems that implement components of mental spatial transformations and explore their properties.

Two classes of mental spatial transformation may be particularly important for everyday spatial reasoning. Object-based spatial transformations are imagined rotations or translations of objects relative to the spatial reference frame of the environment. Egocentric perspective transformations are imagined rotations or translations of one's point-of-view relative to that ref- erence frame. Imagine an experienced surgeon teaching a new resident an endoscopic procedure. In order to decide what the resident should do, the surgeon needs to imagine possible movements of the surgical tools (an object-based transformation). To explain this to the resident, the surgeon needs to imagine what the situation looks like from the resident's point of view (an egocentric perspective transformation). In both cases, relations between environment-centered, object-centered, and viewer-centered representations are updated. However, the nature of the updating differs. F or object-based transformations, an object-based reference frame changes relative to the environment-centered and viewer-centered frames. F or egocentric perspective transformations, the viewer-centered frame changes relative to the environment-centered frame and the object-centered frame, but those two remain fixed relative to each other. These two classes of transformation are by no means exhaustive; others such as imagined limb movements likely play an important role in some spatial reasoning tasks.

Although these two classes of transformation are logically dissociable, it does not follow from this that they are dissociated in the brain or in behavior. One possibility is that different logical classes of spatial transformation are carried out by a single spatial transformation system. Another possibility is that there are specialized neural structures for performing different spatial updating computations. The research reported here was motivated by the latter view, which we call the multiple systems framework (Zacks et al., in press). This framework implies that though similar spatial reasoning tasks may share a number of computational components (stimulus encoding, image comparison, response planning and execution), they should dissociate systematically based on the type of transformation performed. As will be seen in the following paragraphs, the limited evidence available from behavioral experiments, neuropsychological case studies, and neuroimaging studies is consistent with this view.

There is now a large behavioral literature on transformations of spatial mental images. Much of this literature has concentrated on a particular objectbased transformation: mental rotation (for reviews, see 
Kosslyn, 1994; Shepard and Cooper, 1982). This research has demonstrated that observers can solve many kinds of spatial judgment problems by mentally rotating an image of a stimulus. In the most common paradigm, participants judge whether two objects presented at differing orientations are identical or mirror-images. Response time typically increases monotonically with orientation disparity (Shepard and Cooper, 1982; Shepard and Metzler, 1971). Similar results have been observed in paradigms in which participants name novel pictures of common objects. Many objects have a canonical orientation, and naming time for novel pictures increases monotonically as the picture is rotated from that orientation ( $\mathrm{d}$ olicoeur, 1985). These results support the view that mental rotation is a continuous transformation that is isomorphic with physical object rotation, though other accounts have been proposed (J ust and Carpenter, 1985; Pylyshyn, 1979).

Egocentric perspective transformations are less well understood behaviorally. They do not seem to follow the same chronometric pattern as object-based transformations. In an elegant study, Parsons (1987) asked observers to make left-right judgments about pictures of bodies with one arm extended, presented at varying orientations. The functions relating orientation of the stimuli to response time differed substantially and systematically from those observed in mental rotation paradigms, suggesting a different transformation was being performed. Moreover, the relationship between orientation and response time in this task correlated well with that observed when participants were instructed to imagine themselves in the position of the depicted figure (an egocentric perspective transformation).

A few studies have compared egocentric perspective transformations and object-based transformations directly elicited by direct instruction. Huttenlocher and Presson (1973) and Presson (1982) asked observers either to imagine an array of objects rotating or to imagine themselves rotating around the array. Participants reported being able to perform both tasks as requested. For both tasks, trials with rotation were slower than trials without rotation, indicating that some sort of transformation was performed. The pattern of responding indicated that the two transformations have different properties: Matching to a picture was easier following imagined rotations of the array, whereas answering questions about the locations of items was easier after imagined rotations of the viewer. (See also Wraga et al., 1999; Wraga et al., 2000.)

In short, prior behavioral evidence is consistent with the view that multiple systems subserve different spatial transformations, but much of this evidence is indirect.

Neuropsychological and neurophysiological evidence suggests that posterior association cortex is important for performing both sorts of mental spatial transformations. Patients with lesions to posterior parietal, temporal and occipital regions have difficulty in wayfinding (Farrell, 1996), reduced scores on tests of spatial intelligence (Warrington et al., 1986), and impaired performance on laboratory tasks requiring spatial transformations (De Renzi, 1982). The literature also hints at a dissociation between object-based transformations and egocentric perspective transformations, though indirectly. I magined rotations of objects appear to be selectively impaired by lesions to the right hemisphere (Ratcliff, 1979), and are performed more efficiently by the right hemisphere in patients whose corpus callosum has been severed as well as in neurologically intact participants (Corballis, 1997; Ditunno and Mann, 1990). Electroencephalographic (Pegna et al., 1997; Pierret et al., 1994; Rosler et al., 1995; Wijers et al., 1989; Yoshino et al., 2000) and functional imaging (Alivisatos and Petrides, 1997; Barnes et al., 2000; Cohen et al., 1996; Harris et al., 2000; Richter et al., 2000; Richter et al., 1997; Rypma et al., 1996; Tagaris et al., 1997; Vingerhoets et al., 2001) studies have found that tasks thought to require mental rotation yield activity in the same areas, though often in the left as well as right hemisphere. Egocentric perspective transformations appear to be particularly dependent on left posterior cortex. In a classic study, Semmes and colleagues asked patients to point to parts of their own bodies based on a diagram, and to walk simple routes based on a map. Both tasks require egocentric perspective transformations, and both were selectively impaired in patients with focal posterior lesions (Semmes et al., 1963). Recently, functional imaging studies have found activity in left-lateralized posterior regions associated with several tasks that may involve egocentric perspective transformations (Aguirreand D'Esposito, 1997; Bonda et al., 1996). One challenge in interpreting these data, particularly in the case of patient studies, is that the tasks used include substantial stimulus encoding, judgment, and response formulation components, and these are difficult to control adequately.

Zacks et al. (1999) directly compared object-based transformations and egocentric perspective transformations using functional magnetic resonance imaging (fMRI). They adapted a task developed by Ratcliff (1979) in a clinical study, in which participants made spatial judgments about pictures of a schematic human figure with one arm outstretched. In a control condition, participants reported on which side of the screen the outstretched arm of an upright figure appeared. In one experimental condition participants reported which arm of an upright figure was outstretched. They reported solving this problem by imaging themselves in the position of the figure, an egocentric perspective transformation. Compared to the control condition, this led to an increase in activity at the junction of the 
parietal, temporal, and occipital lobes, particularly on the left. In a second experimental condition, participants reported which arm of a figure was outstretched for upside-down figures. Participants reported mentally rotating the figure to upright, an object-based transformation (see also Ratcliff, 1979). This led to further increases in parietal-temporal-occipital cortex, but the further increases were higher, more lateral, and stronger in the right hemisphere. Together, these findings suggest dissociable bases for egocentric perspective transformations and object-based transformations. Similar results have recently been reported in a related paradigm, which included converging data from a split-brain patient (Funnell et al., 2001).

As studies such as these indicate, the human body has a dual status in people's perceptual experience of spatial transformations. On the one hand, people perceive others' bodies moving relative to themselves and to the environmental reference frame. These are real object-based spatial transformations. On the other hand, people also experience their own bodies moving as they locomote. These are real egocentric perspective transformations.

As goes perception, often so goes imagery (Kosslyn, 1994; Podgorny and Shepard, 1978; Shepard, 1994). I magined transformations of bodies may be either object-based spatial transformations or egocentric perspective transformations. Both are important in anticipating changes in the world: object-based transformations for changes in the orientation of other bodies as they move in the world and perspective transformations for changes of point-of-view as we move in the world. The dual status of bodies in invoking both kinds of spatial transformation may provide a window on mental spatial transformations, by allowing one to flexibly elicit different spatial transformations with the same stimuli.

Zacks et al. (in press) explored the ability of bodies to elicit object-based and perspective transformations in a tightly controlled behavioral paradigm. We describe this paradigm in some detail, as it forms the basis for the present study. Two tasks were designed to selectively elicit each type of transformation with the same stimuli and responses, allowing for direct comparisons between the two. The same-different task was designed to elicit an object-based transformation, mental rotation (Shepard and Cooper, 1982; Shepard and Metzler, 1971). Observers viewed pairs of pictures of bodies with an outstretched arm, either identical or mirror images, that differed in their picture-plane orientation, and judged whether they were identical. In the version used by Zacks et al. (and unlike most mental rotation studies) one of the two pictures always appeared at an upright (canonical) orientation. In the left-right task, observers viewed a single picture appearing at a random picture-plane orientation, and identified whether the body's left or right arm was outstretched. In both tasks an object appeared in a left or right version on each trial, in a randomly-chosen orientation. The tasks used identical pictures, and in both cases responses were made by pressing one of two buttons.

The same- different task was expected to yield an object-based transformation, because the task requires comparisons between multiple objects relative to their intrinsic reference frames and can be made without a change to the observer's egocentric perspective. The left-right task was expected to yield an egocentric perspective transformation, because the spatial judgment is specified relative to the reference frame of the pictured body, and can be performed by imagining one's own body in the position of the picture. This hypothesized difference in spatial transformations was predicted to lead to systematic differences in behavioral performance. Based on prior research, if the samedifferent task did in fact elicit an object-based transformation, it should lead to a monotonic relationship between stimulus orientation and response time (Shepard and Metzler, 1971). By contrast, if the leftright task led to an egocentric perspective transformation, prior research indicated that there would be little relationship between orientation and response time (Parsons, 1987). Next, if the two tasks elicit different spatial transformations, they should have different relationships to psychometric tests of spatial ability. Both predictions were borne out by the data.

The results from this paradigm provide independent evidence for the proposal that object-based transformations and egocentric perspective transformations are performed by dissociable neural systems. They also establish that the two tasks, when performed with pictures of bodies, allow an experimenter to selectively elicit object-based and egocentric perspective transformations. The same- different and left-right tasks have two attractive features for neuroimaging studies. First, they allow one to compare putatively different mental spatial transformations while holding constant the stimuli presented and the response required. This is critical, because any experimental task brings to bear a number of sources of neural activity in addition to those of interest. Tight comparisons between tasks that share encoding and response components allow stronger inferences about the source of differences in brain activity between the tasks. Second, these two tasks allow one to parametrically modulate the orientation of the stimuli, which modulates response time in the same- different task but leaves response time in the left-right task unchanged.

The available evidence points toward a model of object-based and egocentric perspective transformations, which can be formulated within the multiple systems framework. The key feature of the model is that updating of the relationship between an object's intrinsic spatial reference frame and those of the environment and the viewer (object-based image transformation) is 
dissociable from updating of the relationship between an observer's egocentric reference frame and those of the environment and the objects within it (egocentric perspective image transformation). This implies that if a task that is performed primarily by object-based transformations is compared to a task performed primarily by egocentric perspective transformations, the regions specific to performing image updating should doubly dissociate. However, object-based spatial transformation tasks and egocentric perspective transformation tasks should also activate a number of common brain areas, because they share a number of operations: stimulus encoding, property lookup, image comparison, response selection, and response execution. Thus, in tightly controlled comparisons one should observe a large number of common active regions and a small number of regions that show differential activity for each of the two classes of transformation.

In the current study, we directly compared neural activity during performance of the same- different and left-right tasks with fMRI. For both tasks pictures of bodies were used, in an attempt to selectively elicit object-based transformations and egocentric perspective transformations, respectively. A rapid event-related design (Buckner and Braver, 1999) was used in which multiple trials of each task were performed, closely spaced, and stimulus orientation was varied from trial to trial. This allowed us to measure changes in brain activity as a function of task, stimulus orientation, and response time.

The study was designed to address three hypotheses regarding object-based and egocentric perspective transformations. First, it was hypothesized that regions in posterior parietal, superior temporal, and occipital cortex would be active during these tasks and modulated by task parameters. Second, it was hypothesized that a subset of these areas would show differential activity in the two tasks, some showing greater activity when performing the same- different task, and others showing greater activity when performing the left-right. Finally, we predicted that areas showing differential activity in the two tasks would be lateralized, with areas more active in the same- different task tending to fall in right posterior regions, and areas more active in the left-right task tending to fall in left posterior regions.

The ability to manipulate task parameters and correlate their behavioral and neural consequences provides a means of further constraining interpretations of functional neuroimaging data. It is now well known that there are areas of the cortex that show increases (Shulman et al., 1997) or decreases (Shulman et al., 1997) across a wide range of cognitive tasks with visual stimuli. Common decreases in activity have been argued to reflect ongoing processing that is transiently interrupted when attention is focused on a task (Gusnard and Raichle, 2001; Raichle et al., 2001). Such processing may include monitoring of one's somatic state and of peripheral sensory input. Performing a task that requires a narrow attentional focus may lead to inhibition of these ongoing functions. When, in any particular study, activity is observed in these areas that doesn't submit to ready explanation, it is tempting to attribute this activity to such ongoing processing. Parametric manipulations provide a more principled way to make such attributions: Changes in activity that are unrelated to the specifics of task performance are unlikely to be affected by parametric task manipulations.

\section{MATERIALS AND METHODS}

\section{Participants}

The research reported here was approved by the human studies committee of the Washington University Medical School, and was carried out in accord with its procedures. Twenty-four participants (15 female, ages 19 to 31) took part. All were right-handed. Data from 6 were participants unusable due to equipment difficulties (3), poor task performance (1), or movement during scanning (2), leaving 18 whose data were analyzed.

\section{Stimuli and Tasks}

Participants made judgments about line drawings of human bodies (see Fig. 1). Each picture showed a man facing the viewer with one arm extended and the other at his side. The orientation of the body in the picture plane was varied in $30^{\circ}$ increments from -180 to $180^{\circ}$ clockwise from upright. Two poses were used: the man's outstretched arm was either extended straight away from the body, perpendicular to the axis of the torso and in the same plane as the rest of the body or crossed over his chest.

In the same- different task, two pictures were presented on each trial. The top picture was always presented in the upright $\left(0^{\circ}\right)$ orientation; which arm was extended (left or right) and which pose was shown (arms crossed or uncrossed) was chosen at random. The bottom picture appeared at a randomly chosen orientation. The pose was always the same as for the top picture, but which arm was extended was chosen at random. Thus, on half of the trials the two figures were identical, and on half of the trials they were mirror images. On each trial the orientation disparity between the two figures varied randomly. Participants were instructed to press a button with one index finger if the two figures were identical (same) and to press a button with the other index finger if the two figures were mirror images (different). Assignment of buttons to same and different responses was counterbalanced across participants. They were told to respond quickly and accurately. 

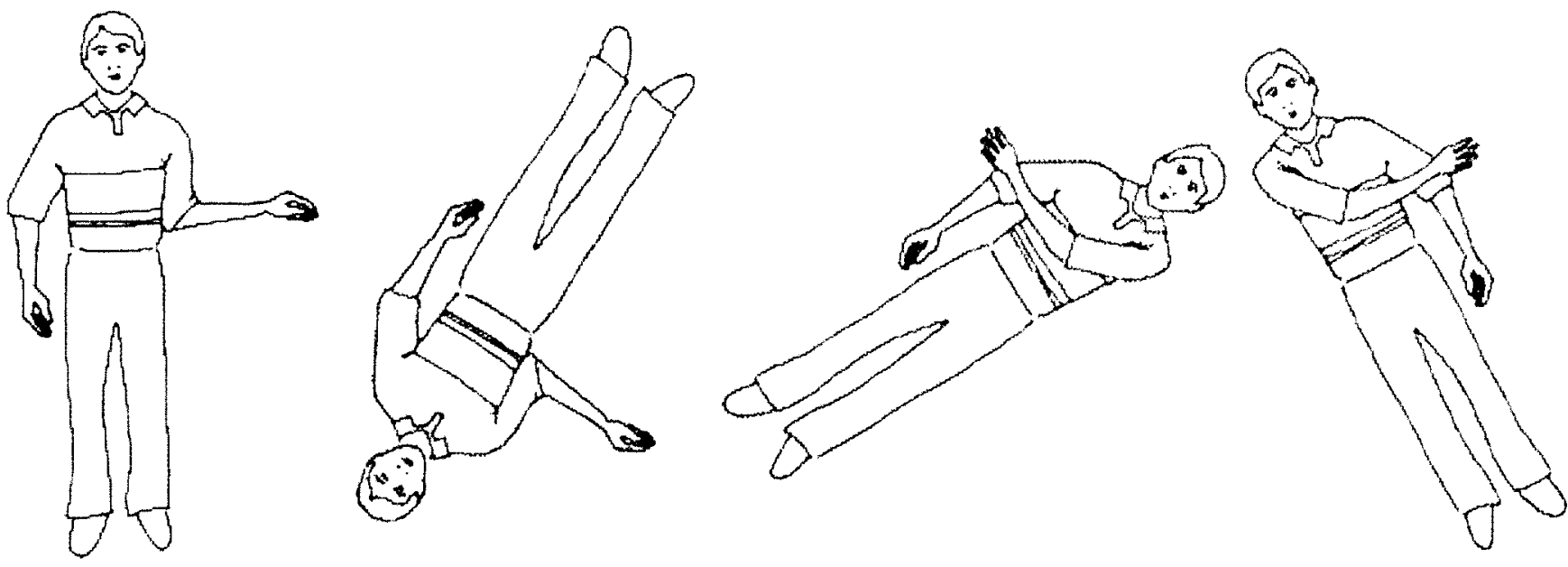

FIG. 1. Examples of stimulus pictures. The first (leftmost) depicts an upright ( 0 degree) figure with his left arm extended straight from the body. The second depicts a figure rotated $-150^{\circ}$, with his right arm extended straight from the body. The third depicts a figure rotated $60^{\circ}$, with his left arm crossed across the body. The fourth depicts a figure rotated $-30^{\circ}$, with his right arm crossed across the body.

In the left-right task, the two pictures presented were identical in all respects. Participants were told they could make their judgment about either figure. The picture's orientation, pose, and which arm was extended were randomly selected on each trial. Thus, the displays for the left-right task were essentially the same as those for the same- different task except that the two figures were al ways identical and the orientation of the top figure varied, as well as that of the bottom figure. Participants were instructed to press a button with their left index finger if the figure's left arm was extended, and to press a button with their right index finger if the figure's right arm was extended. In this task, the second figure is redundant; it was presented to control the visual properties of the stimuli presented. Participants were told to respond quickly and accurately.

F or both tasks, the stimulus remained on the screen for $2.44 \mathrm{~s}$ (one image acquisition). Between trials, a small black plus sign was projected on the middle of the screen, and participants were instructed to fixate it. The duration of the intertrial interval varied randomly (see MRI I maging below).

Displays were rear projected from an LCD projector placed behind the bore of the scanner to a screen placed at the opening of the scanner, and were viewed through a mirror attached to the head coil. The stimuli took up approximately $8^{\circ}$ of visual angle in the horizontal direction, and $16^{\circ}$ in the vertical direction. Responses were recorded using a custom built fiber optic button box, placed on the participants abdomen with one index finger on each of two buttons. Stimulus presentation and response recording were controlled by a Macintosh computer (Apple, Cupertino, CA) running the PsyScope experimental presentation software (Cohen et al., 1993).
Participants performed two alternating blocks of each task. Task was blocked because switching between the two tasks on each trial was found to be difficult. Each block consisted of 56 trials. Across the two blocks of the same- different task, all possible combinations of pose (2), extended arm (2), orientation of the bottom figure (12), and match between the top and bottom figure (2) were tested once. Across the two blocks of the left-right task, all possible combinations of pose (2), extended arm (2), and orientation (12) were tested twice. (F or data analysis, clockwise and counterclockwise rotations were combined, i.e., $30^{\circ}$ was grouped with $-30^{\circ}, 60^{\circ}$ with -60 , etc. We therefore tested 0 and $180^{\circ}$ twice as often as the other orientations, to maintain equivalent numbers of observations at each level of collapsed orientation.) Before scanning, participants were given instructions and practiced 56 trials of each task outside the scanner.

\section{Magnetic Resonance I maging}

Imaging was performed on a $1.5 \mathrm{~T}$ Vision scanner (Siemens, Erlangen, Germany) at the Research Imaging Center of the Mallinckrodt I nstitute of Radiology at Washington University. Structural images were acquired using a sagittal 3-D magnetization prepared rapid acquisition gradient echo recalled (MP-RAGE) T1-weighted sequence, with $1-\mathrm{mm}^{3}$ isotropic voxels. Functional imaging was performed using an asymmetric spin-echo echoplanar pulse sequence with a flip angle of $90^{\circ}$ and a time to echo of $37 \mathrm{~ms}$, optimized for blood oxygen level dependent (BOLD) contrast (T2*) (Conturo et al., 1996; Ogawa et al., 1990), which has been shown to be closely related to local synaptic activity (Logothetis et al., 2001). Eighteen axial slices were acquired with a thickness of $7 \mathrm{~mm}$ and in-plane 
resolution of $3.75 \mathrm{~mm}$. The time to recall (TR) for each slice was $135.2 \mathrm{~ms}$, resulting in a total acquisition time of $2.44 \mathrm{~s}$ for each functional image. The time to echo (TE ) was $37 \mathrm{~ms}$ and the flip angle was $90^{\circ}$. T2-weighted structural images were acquired in the planes of the functional images, with an in-plane resolution of 0.938 $\mathrm{mm}$ to facilitate alignment of the functional data to a standard stereotactic space.

Each functional run took 488 s (200 image acquisitions), and included one block of 56 trials of one of the two tasks. The first four images were acquired before beginning the task to allow transient signals to diminish. Presentation of the stimulus was time locked to the beginning of the image acquisition, and the stimulus remained on screen for one image acquisition (2.44 s). Stimulus presentation was followed by a variable intertrial interval, during which the participant fixated on a small central cross (see Stimuli and Tasks). On half of the trials this interval was $0 \mathrm{~ms}$, i.e., the next trial began immediately. On one quarter of the trials the interval was $2.44 \mathrm{~s}$ (one image acquisition), and on one quarter of the trials it was $4.88 \mathrm{~s}$ (two image acquisitions). This approximates an exponential distribution of intertrial intervals, and has been shown to optimize statistical power for event-related fMRI designs (Ollinger et al., 2001).

Head motion was minimized by fitting each participant with a thermoplastic mask after placement on the scanner bed. Participants were briefed regarding the importance of minimizing head motion and were re minded of this regularly.

\section{fMRI Data Analysis}

Functional data were preprocessed prior to statistical analysis using methods standard for our laboratory (Ojemann et al., 1997; Zacks et al., 2001). First, individual images for each scan were collated into a single four dimensional array. Second, timing offsets among slices were compensated for using sinc interpolation. Third, systematic odd vs. even intensity differences due to contiguous interleaved slice acquisition were removed using suitably chosen scale factors. Fourth, head motion was corrected using a six parameter rigid body realignment with 3-D cubic spline interpolation. Finally, the MP-RAGE image and functional data were aligned to an atlas constructed by the methods of Lancaster et al . (2000) to conform to the coordinate scheme of Talairach and Tournoux (1988).

The data were analyzed as a function of task and response time, using in-house software (Ollinger et al., 2001). Although orientation was the experimentally manipulated variable, the behavioral pattern observed in prior studies of the two tasks dictated an analysis in terms of response time rather than orientation, be cause responses were expected to be slower and more dependent on orientation in the same-different task.
This means that in an analysis based on orientation, effects of task and orientation would be confounded by response time. Response times for correct responses were binned on a within-participants basis into three tertiles: fast, medium, and slow. Bins were calculated across both tasks, which controls for overall difficulty differences between the two tasks, but results in different numbers of trials for each combination of task and response time. The mean value for the first tertile (33.3rd percentile) was $1028.9 \mathrm{~ms}, \mathrm{SD}=154.4 \mathrm{~ms}$; the mean value for the second tertile (66.7th percentile) was 1320.7, SD = $198.1 \mathrm{~ms}$. For both analyses, seven fMRI data acquisition timepoints (each taking up $2.44 \mathrm{~s}$, for a total time window of $17.08 \mathrm{~s}$ ) following stimulus presentation were included in each trial. MR signal was modeled as a function of timepoint as well as task and response time, rather than by fitting an hemodynamic response function to the data. This approach has the advantage of avoiding assumptions about the shape of the hemodynamic responses in any given brain region (Burock and Dale, 2000). Thus, for both analyses the experimental design was a 2 (task) by 3 (response time) by 7 (timepoints) repeated measures design.

Analysis proceeded in three stages. First, activity related to task performance in each voxel of each participant's brain was modeled using the general linear model. Second, these modeled responses were submitted to a repeated measures analysis of variance (ANOVA), and regions that showed task-related transient changes were identified. Third, activity in those regions was characterized using regionwise repeated measures ANOVAs.

In the first stage, evoked brain responses for each trial typefor each voxel of each participants brain were estimated using the general linear model with the MR signal at each voxel during each image acquisition as the dependent variable. The model included predictor variables for task, orientation or response time, and timepoint. Low frequency noise and linear drift were also modeled and removed at this stage, and the data were spatial smoothed with a gaussian kernel, full width at half maximum $=7.5 \mathrm{~mm}$.

In the second stage, these modeled responses were used as the dependent measure in a voxel-wise repeated measures analysis of variance (ANOVA), with task, response time, and timepoint as independent variables. This resulted in an $\mathrm{F}$ statistic for each voxel for each main effect and interaction. The degrees of freedom for the $F$ statistics were adjusted to correct for inhomogeneous variance (Ollinger and McAvoy, 2000), the map of the main effect of timepoint was converted from $F$ to $Z$ statistics, and regions of reliable activity were identified by selecting voxels within a contiguous cluster of five or more face-connected voxels whose $Z$ score exceed 4.5. (Henceforth, we will refer to voxels or regions showing reliable task-related changes in $\mathrm{fMRI}$ 
Same-different task

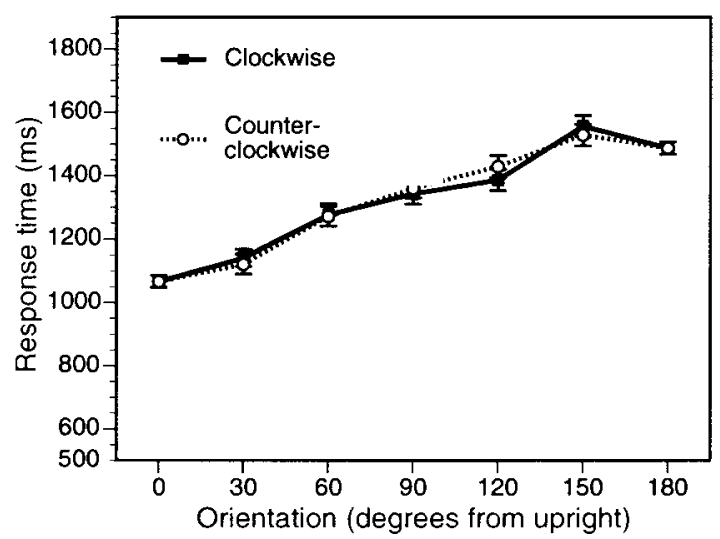

Left-right task

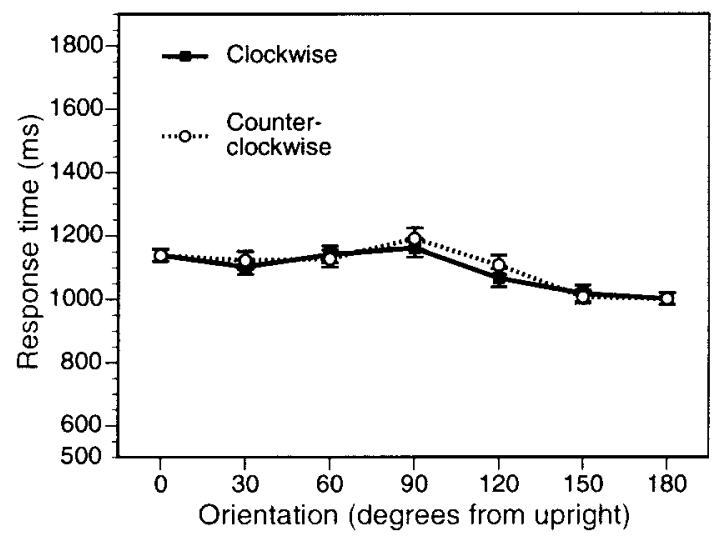

FIG. 2. Relationship between orientation and response time for the two tasks. Error bars depict standard errors of the mean.

signal as "activated.") This threshold has been shown to correspond to a mapwise type I error rate of 0.05 (McAvoy and Ollinger, 2001). The result of this stage was a map of voxels that in the group showed reliable transient changes associated with task performance for the group as a whole.

In the third stage regions were selected from this map of activated voxels by choosing peaks in which the $Z$ statistic was at a local maximum. To identify peaks, the activation map was smoothed with a 6-mm hard spherical kernel and peaks were chosen by an automatic al gorithm. Peaks closer than $18 \mathrm{~mm}$ were combined by taking the midpoint between them. For each peak, a $12-\mathrm{mm}$ sphere was drawn around the peak voxel; overlapping spheres were divided at the plane midway between the peaks. Activated voxels within each sphere were averaged to form a region. The averaged timecourses from these regions were then submitted to a regionwise ANOVA with the same independent variables as the original voxel wise ANOVA. This allowed us to characterize, for each region identified on the basis of the main effect of timepoint, whether it showed a reliable main effect of task, of response time, or reliable interactions between the main effects. Note that under the null hypothesis of no effects each of the main effects and interactions are independent. This means that the identification of regions based on the main effect of timepoint and subsequent probing of their other main effects and interactions with regionwise analyses is unbiased. The regionwise ANOVAs invol ved a substantial number of comparisons, so Bonferroni corrections were used (i.e., the nominal type I error rate of 0.05 was divided by the number of regions analyzed).

\section{RESULTS}

Behavioral Performance

Participants whose error rate was higher than $25 \%$ in any block were excluded from further analysis; one participant was replaced based on this criterion (see Participants above). For the remaining participants, performance was accurate: Error rates were $3.2 \%$ in the left-right task and $5.0 \%$ in the same- different task. There were also a small number of trials on which no response was recorded, either because the participant failed to respond or because the button box failed (2.8\% in the left-right task and $3.7 \%$ in the samedifferent task).

As can be seen in Fig. 2, performance in the two tasks differed substantially. In the same- different task, response time increased monotonically with stimulus orientation, whereas in the left-right task response time was independent of orientation. This pattern was tested first by calculating the mean response time for each participant for each combination of task and orientation, and submitting these scores to a 2 (task) by 12 (orientation) repeated-measures ANOVA. There were reliable main effects of task $[F(1,30)=$ $14.0, \mathrm{P}<0.001]$ and orientation $[\mathrm{F}(11,165)=10.8, \mathrm{P}<$ $0.001]$, as well as a reliable task-by-orientation interaction $[F(11,165)=25.3, P<0.001]$. To characterize the relationship between orientation and response time better, we calculated the correlation between orientation and response time for correct trials on each task for each participant. The mean correlation for the same- different task was $0.48(S D=0.10)$; the mean correlation for the left-right task was -0.18 (SD = $0.18)$. These two means differed reliably, $\mathrm{t}(15)=12.2$, $\mathrm{P}<0.001$. Both distributions of correlations differed reliably from zero. For the left-right task, $\mathrm{t}(15)=4.17$, $\mathrm{P}<0.001$; for the same- different task, $\mathrm{t}(15)=19.0$, $\mathrm{P}<0.001$.

The pattern of increasing response times with increasing orientation of the same- different task, but not for the left-right task, is consistent with that observed in behavioral studies with much larger samples (Zacks et al., in press) In the current dataset, the correlation between orientation and response time for 


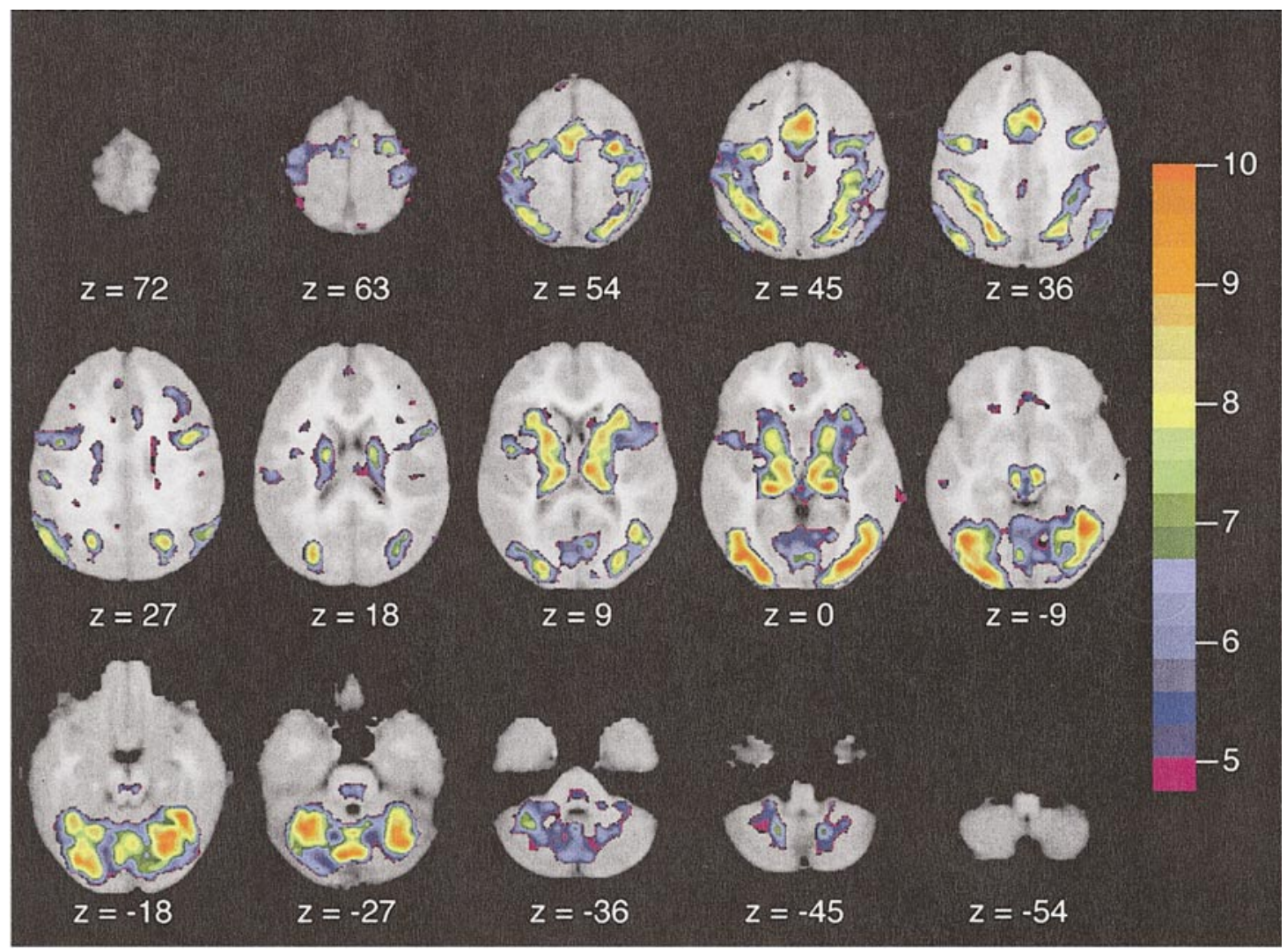

FIG. 3. Regions of the brain that showed task-related activity, superimposed on the average anatomical image for the 16 participants. Highlighted regions had a corrected $Z$ score greater than 4.5 and were contained in a region of at least five such voxels, a threshold which has a mapwise type I error rate of $P=0.05$ (see fMRI Data Analysis).

the left-right task was slightly but reliably negative, which has not been observed in previous studies. The difference in response time profiles between the two tasks is consistent with the view that they involve different spatial reasoning subsystems.

\section{Identifying Regions of Transient Activity}

Voxels that showed transient activity when performing the spatial reasoning tasks were identified as described in fMRI Data Analysis above. A voxelwise ANOVA was conducted, with timepoint relative to stimulus onset, task, and response time as independent variables and MR signal as the dependent variable. Voxels that showed a main effect of timepoint above threshold $(Z=4.5)$ and were in a contiguous cluster of seven or more voxels were identified and are shown in Fig. 3. As expected, a considerable volume of tissue showed reliable task-related activity. Clusters of activity included primary visual cortex, motor cortex, the cerebellum, and a significant portion of the parietal and posterior temporal lobes. Voxels identified at this stage could reflect activity due to low-level visual processing of the stimulus, the execution of a motor response, or any other neural change involved in per- forming the task, in addition to activity due to the performance of a mental spatial transformation. Peaks of most reliable task-related modulation within the activated voxels were identified and regions of interest were drawn around those peaks, using automated algorithms (see fMRI Data Analysis, above). The algorithms identified 36 peaks, spanning the large volume of activated tissue. The locations of these peaks are given in Table 1. Estimated timecourses of activity were averaged over each region for each participant, and each region was submitted to a 2 (task) by 3 (response time) by 7 (timepoint) ANOVA.

In the regionwise ANOVA, regions whose activity was related to the participant's response time will show a main effect of response time or a response time-by-timepoint interaction. Of the 36 regions that showed task-related activity, 32 showed a reliable response time-by-timepoint interaction. Of the 32, 5 also showed a reliable main effect of response time (see Table 1). (There were no regions that had a reliable main effect of response time but no interaction.) The typical pattern is illustrated in Fig. 4. For both tasks, slow trials showed greater activity than fast trials; with medium trials falling in between. 
a

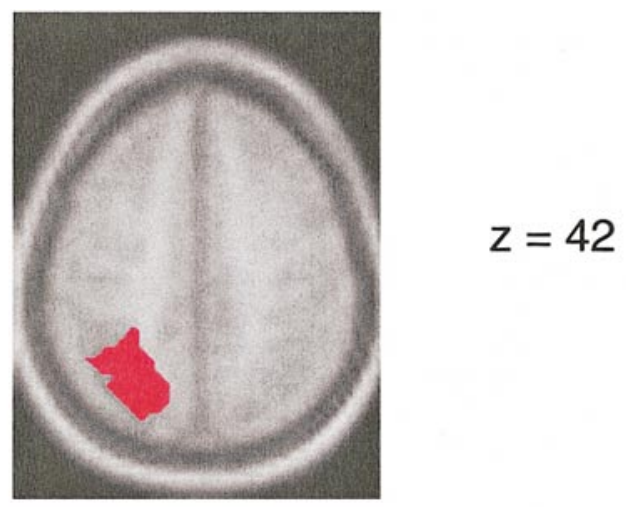

b
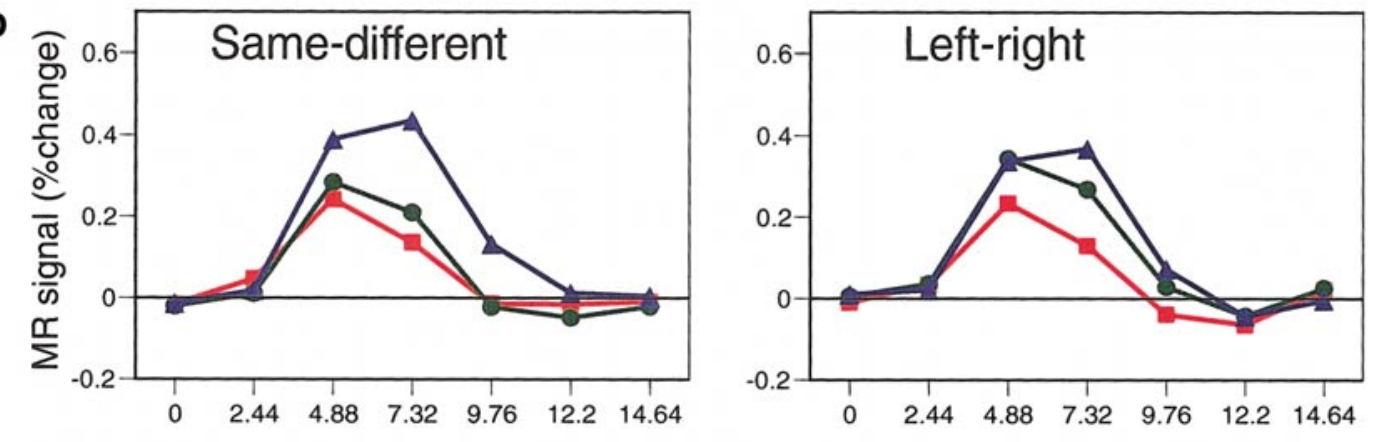

Time (s)
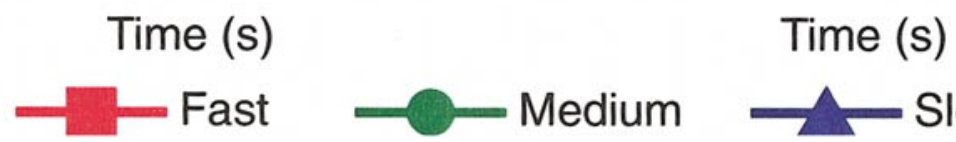

Medium

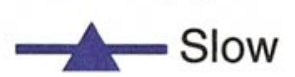

FIG. 4. Timecourse of one typical brain region whose activity was modulated by response time. The region shown here is number 33 in Table 1. This region had a reliable main effect of response time and reliable interaction between response time and timepoint in the regionwise ANOVA (see fMRI Data). An axial slice through the center of the region is shown in a. (b) Graphs the average timecourse of the region for fast, medium, and slow trials for each task. In both tasks, greater activity was observed for slower trials.

Regions Whose Activity Was Modulated by Task

In the regionwise ANOVA, regions whose activity profile was different for the two tasks will exhibit a task by timepoint interaction or a main effect of task. Three regions showed a reliable task by timepoint interaction (see Fig. 5a and Table 1). The most superior of these (region 19 in Table 1) spanned the occipitotemporal junction and middle occipital gyrus (BA 18/19/ 37). The two inferior regions included the posterior lingual gyrus (BA 18/19) and superior medial cerebellum. All regions showed transient (i.e., task-related) increases for both tasks, and larger changes in activity for the same- different task than the left-right task. That is, for every region in which activity differed for the two tasks, the difference was that the change in activity was greater for the same- different task. The typical pattern is shown in Fig. 5b. There were no regions that showed a reliable main effect of task.

Decreases in Activity and Lack of Modulation by Response Time

Inspection of the timecourses showed that most regions were characterized by an increase following stim- ulus onset that closely resembled the hemodynamic response to a brief impulse of neural activity (Boynton et al., 1996; Miezin et al., 2000; see Fig. 4). However, seven regions showed decreases, rather than increases, in activity. These are shown in Fig. 6 (see also Table 1), and included posterior lateral parietal cortex, anterior cingulate cortex, the precuneus, and medial prefrontal cortex. This network has been shown to consistently decrease in blood oxygenation during a wide range of cognitive tasks (Shulman et al., 1997), and may reflect temporary inhibition of a default high level of activity in these regions (Gusnard and Raichle, 2001; Raichle et al., 2001). Activity in these regions was relatively insensitive to changes in task or in the speed of response. N one of the regions had a reliable main effect of task or task-by-timepoint interaction. The three parietal regions showed reliable response time-by-timepoint interactions $[F(12,180)>=2.91$, corrected $P<=0.04]$, but these were small compared to the regions that increased in activity during task performance (see Table 1).

As noted previously, 32 of the 36 activated regions were modulated by response time, as indicated by an interaction between response time and timepoint. All 
TABLE 1

Local Peaks of Brain Activity during Spatial Transformation Tasks

\begin{tabular}{|c|c|c|c|c|c|}
\hline Region & $\begin{array}{l}\text { Atlas coordinate } \\
\text { (Talairach and } \\
\text { Tourmoux, 1988) }\end{array}$ & Location & $\begin{array}{l}\text { Response time } \\
\text { by timepoint }\end{array}$ & $\begin{array}{l}\text { Task by } \\
\text { timepoint }\end{array}$ & $\begin{array}{c}\text { Decrease from } \\
\text { baseline }\end{array}$ \\
\hline 1 & $-19,-54,-44$ & L. cerebellum & $* * *$ & & \\
\hline 2 & $22,-50,-43$ & R. cerebellum & $* * *$ & & \\
\hline 3 & $03,-32,-27$ & Pons & $*$ & & \\
\hline 4 & $-30,-56,-26$ & L. cerebellum & $* * *$ & & \\
\hline 5 & $-02,-73,-26$ & Med. cerebellum & $* * *$ & & \\
\hline 6 & $36,-59,-16$ & R. cerebellum & $* * *$ & & \\
\hline 7 & $-56,-29,-13$ & L. mid. temporal gyrus & & & Yes \\
\hline 8 & $-32,-82,-06$ & L. inf./mid. occipital gyri (BA 18) & $* * *$ & & \\
\hline 9 & $-01,31,-05$ & Ant. cingulate gyrus & & & Yes \\
\hline 10 & $-15,-69,-03$ & L. lingual gyrus (BA 18/19) & $* * *$ & $*$ & \\
\hline 11 & $40,-74,00$ & R. occipitotemporal sulcus (BA 19/37) & $* * *$ & & \\
\hline 12 & $-14,-25,02$ & L. thalamus & $* * *$ & & \\
\hline 13 & $13,-25,03$ & R. thalamus & $* * *$ & & \\
\hline 14 & $08,-74,04$ & R. lingual gyrus (BA 18) & $* * *$ & $*$ & \\
\hline 15 & $-45,-02,05$ & L. sylvian fissure & $* * *$ & & \\
\hline 16 & $22,06,06$ & R. putamen & $* * *$ & & \\
\hline 17 & $-23,07,07$ & L. putamen & $* * *$ & & \\
\hline 18 & $-24,-77,18$ & L. mid. occipital gyrus (BA 18/19) & $* * *$ & & \\
\hline 19 & $30,-74,19$ & R. occipitotemporal junction (BA 18/19) & $* * *$ & $*$ & \\
\hline 20 & $48,-24,20$ & R. sylvian fissure & $* * *$ & & \\
\hline 21 & $-03,43,22$ & Med. frontal gyrus, cingulate gyrus (BA 9/32) & & & Yes \\
\hline 22 & $-54,-25,23$ & L. sylvian fissure & $* *$ & & \\
\hline 23 & $34,30,25$ & R. mid. frontal gyrus (BA 9/46) & $* * *$ & & \\
\hline 24 & $-47,-69,31$ & L. angular gyrus (BA 19/39) & $*$ & & Yes \\
\hline 25 & $-59,-52,33$ & L. supramarginal gyrus (BA 40) & * & & Yes \\
\hline 26 & $51,-63,33$ & L. angular gyrus (BA 19/39) & $*$ & & Yes \\
\hline 27 & $-45,-05,35$ & L. precentral gyrus (BA 6) & *** & & \\
\hline 28 & $23,-66,40$ & R. precuneus (BA 19) & $* * *$ & & \\
\hline 29 & $02,-30,41$ & Cingulate gyrus (BA 31) & & & Yes \\
\hline 30 & $36,-04,41$ & R. precentral gyrus (BA 6) & $* * *$ & & \\
\hline 31 & $-44,-36,42$ & L. inf. parietal lobule (BA 40) & $* * *$ & & \\
\hline 32 & $04,09,42$ & Cingulate gyrus (BA 24/32) & $* * *$ & & \\
\hline 33 & $-28,-57,43$ & L. sup. parietal lobule (BA 7/19) & $* * *$ & & \\
\hline 34 & $32,-41,48$ & R. sup. parietal lobule (BA 7) & $* * *$ & & \\
\hline 35 & $-34,-16,53$ & L. pre/post-central gyri & $* * *$ & & \\
\hline 36 & $-01,-06,60$ & Med. frontal gyrus (BA 6) & $* * *$ & & \\
\hline
\end{tabular}

Note. Peaks were identified based on the main effect of timepoint in a voxel wise ANOVA, as described in the text. F or each peak, the results of the regionwise ANOVA are summarized in the third and fourth columns. Modulation by response time is indicated in column 3 (response time by timepoint interaction) and modulation by task is indicated in column 4 (task by timepoint interaction). All P values are corrected for multiple comparisons with a Bonferroni correction.

a The main effect of response time was also statistically reliable $(\mathrm{P}<0.05)$.

$* \mathrm{P}<0.05$.

$* * \mathrm{P}<0.01$.

$* * * \mathrm{P}<0.001$.

four of the regions that did not have a reliable response time by timepoint interaction were areas whose activity decreased during task performance. Further, the three remaining regions in which decreases were observed showed a small relationship to response time (see Table 1). In other words, every region whose activity increased when performing the task also showed modulation by response time, and every region whose activity decreased when performing the task showed weak or no modulation by response time.
There were no regions that showed a reliable threeway interaction between task, response time, and timepoint.

\section{DISCUSSION}

The current results confirm that mental spatial transformations are demanding cognitive operations. The present results show evidence of widespread BOLD activity during performance of these tasks, consistent with previous neuroimaging results. The tasks 
used in the current study require encoding a visual stimulus, which has been found to lead to hemodynamic changes in primary visual cortex (Kwong et al., 1992) as well as extrastriate occipital and temporal areas (De Yoe et al., 1994). Planning and executing a motor response on each trial would be expected to draw on primary motor cortex and motor planning areas (Rao et al., 1993). The tasks also require adaptively controlling attention and formulating a decision, processes known to depend on prefrontal cortex (Casey et al., 2000). Performing two spatial reasoning tasks led to robust activation in all these areas. The statistical methods used here permitted identification of these areas without a priori assumptions about the shape of the hemodynamic response in any of the regions involved.

\section{Effects of Task}

In a small subset of activated regions, all in right posterior cortex, the change in fMRI signal was greater for the same- different task than for the left-right task. Because response time was explicitly modeled in these analyses, this difference can not be attributed to a confound with response time. These areas, then, are differentially involved in performing the same- different task. Regions showing task effects included right parietal, occipital, and temporal cortex. The differential activity of these regions during performance of an object based transformation is consistent with previous imaging results (Barnes et al., 2000; Cohen et al., 1996; Kosslyn et al., 1998; Richter et al., 2000, 1997; Tagaris et al., 1997; Vingerhoets et al., 2001). The right lateralization of that activity is consistent with one recent study of mental rotation of alphanumeric characters (Harris et al., 2000), but inconsistent with another study using a similar method (Alivisatos and Petrides, 1997).

The finding that right hemisphere areas are more active for object-based transformations than egocentric perspective transformations is consistent with that of a recent study that manipulated the stimuli, rather than the task, to investigate spatial transformations. Kosslyn et al. (1998) asked participants to make samedifferent judgments about the original Shepard and Metzler (1971) cube figures and about pictures of hands, both during PET scanning. They observed greater right hemisphere activity for the cube stimuli than for the hands. One possibility is that the hands, being human body parts, led to relatively more use of egocentric perspective transformation strategies. However, this interpretation should taken as speculative, because the use of hands as stimuli probably also elicited substantial imagined limb movements (Parsons, 1987; Parsons et al., 1995). In terms of the multiple systems framework, imagined limb movements constitute a third class of spatial transformation, and so these results are at least as likely to reflect the contribution of that system as the hypothesized egocentric perspective system. (In fact the relationship between movement-related processing and mental rotation was the object of the Kosslyn et al. study.)

The finding that activity in the medial superior cerebellum was modulated by task is something of a surprise. However, few previous studies have imaged the cerebellum during mental transformation tasks. This area has been found to show hemodynamic responses to a wide range of demanding cognitive tasks (Shulman, Corbetta et al., 1997), suggesting a nonspecific role. However, it has also been implicated in recent functional imaging studies of biological motion perception (Grossman et al ., 2000) and the item-specific component of a skill learning task (Poldrack and Gabrieli, 2001). One speculation that is consistent with both of these results is that the activity may be due to the processing of imagined object movement in an objectbased transformation.

Although the visual stimulus presented in the two tasks was equated by using two figures for both the same-different and left-right tasks, this does not guarantee that demands on attention were also equated. The fact that one can perform the left-right task while attending to only one of the figures, whereas performing the same- different task requires comparing the two figures, leads to the prediction that the same-different task would place greater demands on spatial attention. This would lead one to predict that areas known to be involved in planning eye movements and shifts of spatial attention, such as the frontal eye fields and supplementary eye fields, would be more active in the same- different task. Overall, we did observe robust activity in the lateral precentral sulcus and medial frontal gyrus, regions associated with human FEF and SEF, respectively (Corbetta et al., 1998). However, we observed no task differences in these regions, suggesting that differences in spatial attention demands were not dramatic.

\section{Response Time and Neural Duty Cycle}

A majority of activated regions showed greater changes in MRI signal for trials on which latencies were longer. Such a pattern is evidence for a duty cycle effect, in which greater MRI signal during a task may be attributable to the participant's having spent a greater proportion of each trial performing the task (and a smaller proportion resting between trials). In task comparisons based on block designs, duty cycle effects are often a potential confound, because in a block design areas that are equally active during performance of two tasks may appear to be more involved in the task that is performed more slowly. In the current case, effects of response time were directly modeled and are of interest: Greater signal changes in an 


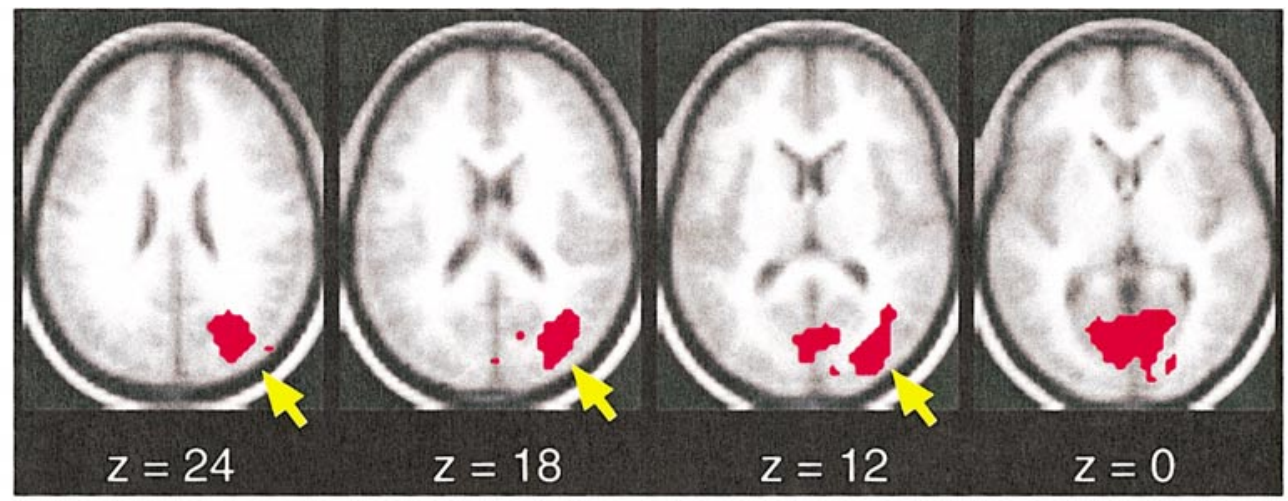

b
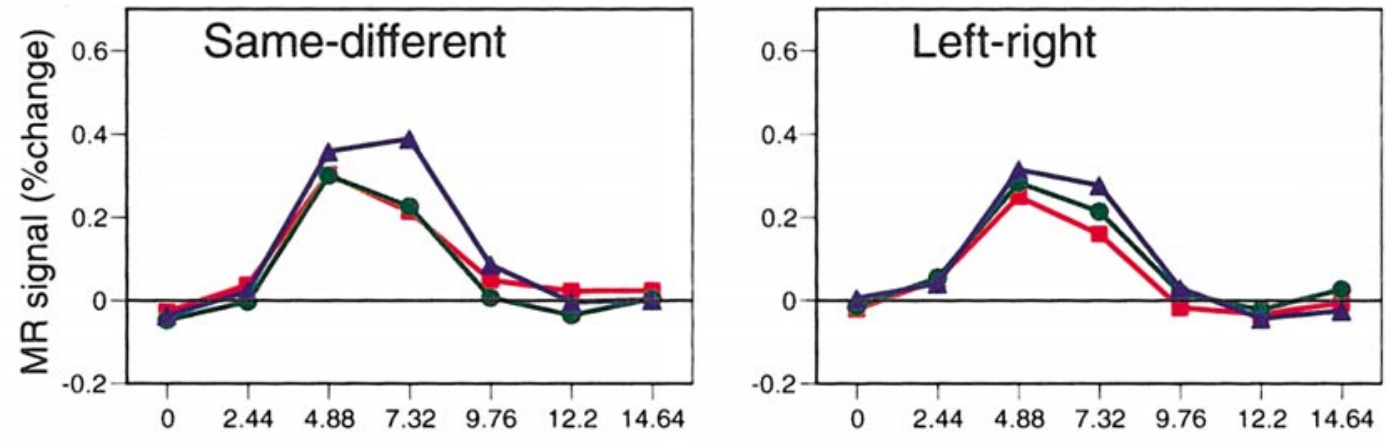

Time (s)
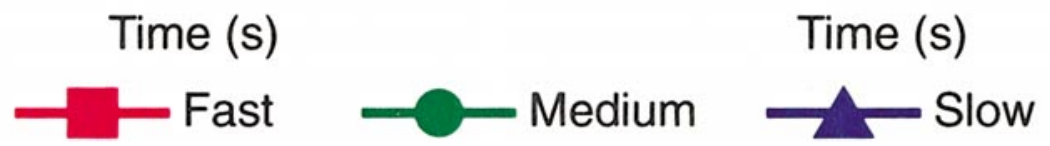

FIG. 5. Regions of the brain whose task-related response differed for the two tasks. (a) Shows the locations regions that had a reliable task by timepoint interaction in the regionwise ANOVA (see Regions whose activity was modulated by task), superimposed on the average anatomical image for the 16 participants. (b) Graphs the average timecourse of one representative region (number 19 in Table 1 , indicated with yellow arrows) for fast, medium, and slow trials for each task.

area for slow trials compared to fast trials indicates the region in question is in some way related to the mental transformation. It does not follow, however, that such a region is responsible for actually implementing the image transformation rather than preparing a response or representing the visual image, because these input and output processes may be prolonged on trials with longer responses.

The minority of regions whose activity was not modulated by response time were regions in which activity decreased while performing the task, possibly reflecting transitory inhibition of processes associated with the brain's "default state" (Gusnard and Raichle, 2001; Raichle et al., 2001). If ongoing monitoring processes are actively inhibited during the performance of a cognitive task, this pattern would be expected. Areas that are active during task performance can show great sensitivity to duty cycle: The longer the task, the more neural activity and the greater the BOLD response. However, active inhibition of ongoing processing is likely to be a coarser grained effect, corresponding to a signal to temporarily suspend activity in response to the onset of a stimulus.
The fact that many regions involved in performance of these tasks show effects of response time is an interesting and somewhat counterintuitive finding. It seems clear that a region involved in the actual computation of an analog transformation should show greater activity on trials for which the transformation takes longer. But primary visual cortex? (Recall that the stimuli remained on the screen for a fixed interval, independent of response time, and participants were instructed to fixate the center of the screen continuously.) Effects of response time in primary sensory areas could arise from increased attention to the stimulus on longer trials, a supposition that is consistent with the finding that participants visually scan stimuli throughout mental rotation studies ( ust and Carpenter, 1985). Several laboratories have now reported attentional modulation of BOLD signals in primary visual cortex (e.g., Gandhi et al., 1999; Huk and Heeger, 2000; Somers et al., 1999). Effects of response time in primary motor cortex could reflect the downstream consequences of motor conflict, given that people appear to prepare multiple motor responses in tasks that generate response conflict (DeSoto et al., 2001). In fu- 
a

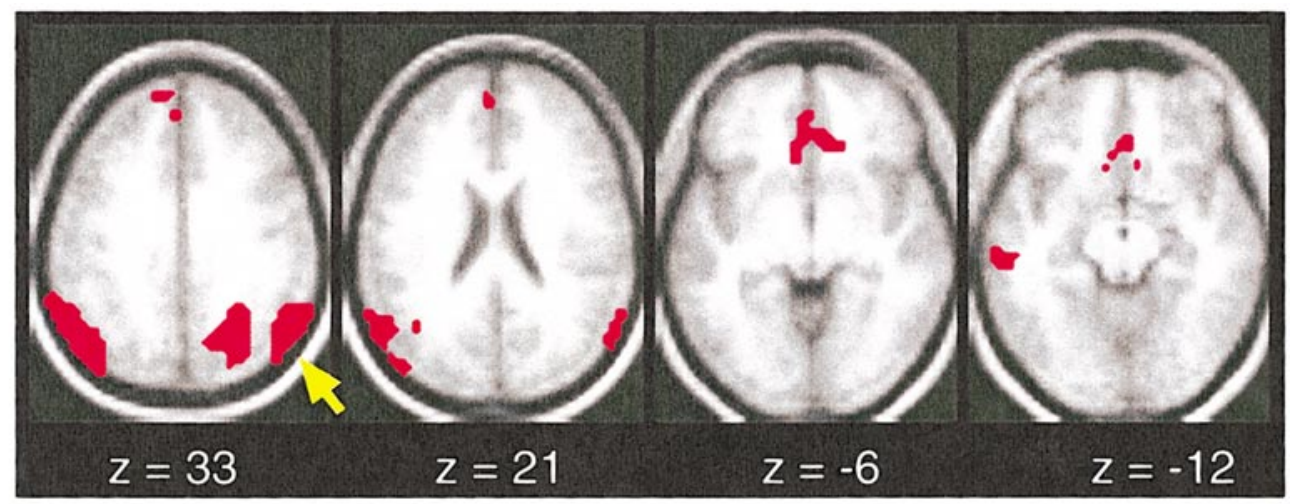

b
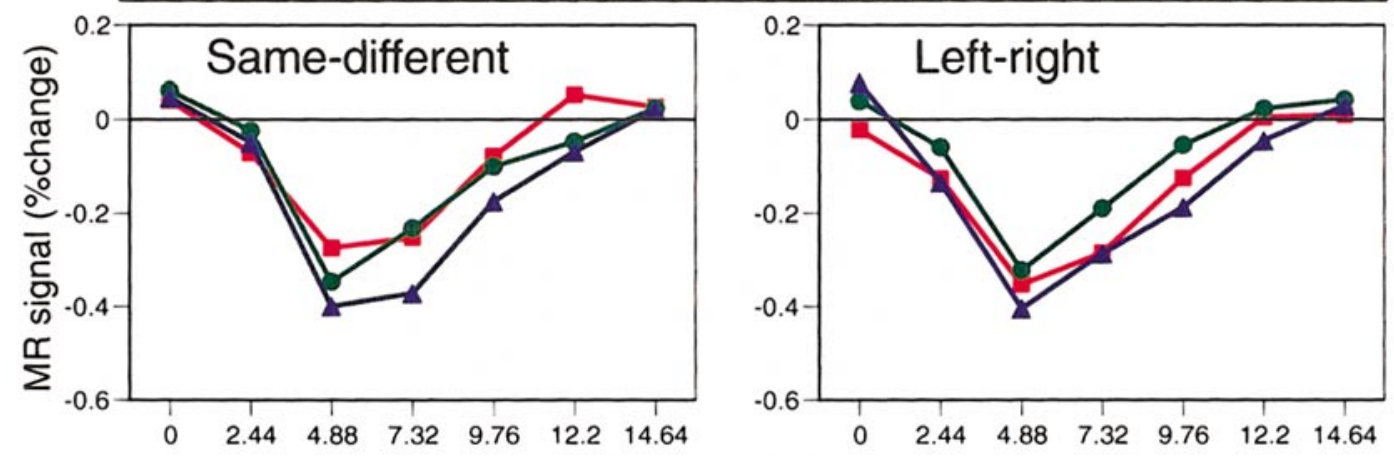

Time (s)

Time (s)
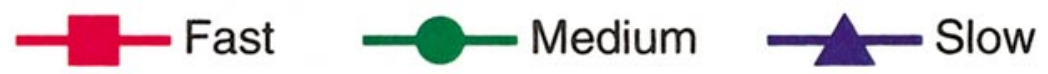

FIG. 6. Regions of the brain that decreased in activity during task performance. (a) Shows the locations of regions for which task performance was associated with a transient decrease inactivity, superimposed on the average anatomical image for the 16 participants. (b) graphs the average timecourse of one representative region (number 26 in Table 1, indicated with a yellow arrow) for fast, medium, and slow trials for each task.

ture research, the effects of attention duration on activity in early visual areas can be explored by limiting stimulus presentation duration. Effects of attention duration, and of motor conflict, could be characterized by developing catch trial designs in which no spatial judgment is performed on some proportion of trials (Ollinger et al., 2001).

\section{Implications for the Multiple Systems Framework}

The data provide partial support for the view that multiple systems subserve different mental spatial transformations. The model described in the Introduction assumed that there are different brain areas responsible for different classes of spatial image updating. One area or set of areas was hypothesized to perform object-based spatial image updating, whereas a different area or set of areas was hypothesized to perform egocentric perspective transformations of the mental image. That is, the model predicts the presence of double dissociations between object-based transformations and egocentric perspective transformations. As predicted, there were regions that showed differential activity in the two tasks. Also as predicted, regions that were more active for the same- different task were all in right posterior cortex. However, no areas were observed that were more active in the left-right task than the same- different task. In short, whereas the model predicts the presence of double dissociations, only a single dissociation was observed.

One possibility is that the failure to observe regions with greater $\mathrm{fMRI}$ signal changes during the left-right task simply reflects a type II error. As conducted here, the left-right task was performed overall faster than the same- different task. This may make areas that are preferentially engaged by the left-right task more difficult to detect. (Note that this is an issue of statistical power, rather than of confounding, which could lead to type I errors.) One way to test this possibility is to design tasks to elicit egocentric perspective transformations that can be behaviorally validated and which are as difficult or more difficult than a comparable task dependent on object-based spatial transformations.

Another possibility is that the proposed model requires revision. The current data are also consistent with a hierarchical version of the model, in which one set of areas is required for egocentric perspective trans- 
formations, and additional processing is required to perform object-based transformations. That is, the computations used to perform an object-based transformation may include the computations used to perform an egocentric perspective transformation as a strict subset.

At this point we favor the original model based on the sum of the available evidence. As discussed in the Introduction, the neuropsychological literature provides indirect evidence for the predicted pattern of dissociation. Moreover, the hierarchical model has a hard time accounting for the fact some spatial judgments are made more easily following an egocentric perspective transformation than following an objectbased transformation (Huttenlocher and Presson, 1973; Presson, 1982; Wraga et al., 2000). According to that model, there should be questions that are difficult to answer after egocentric transformations, because the required information hasn't been computed, but if the object-based transformation contains the egocentric perspective transformation as a subcomponent there should be no questions that are easier to answer after an egocentric perspective transformation. A second behavioral observation also poses difficulty for the hierarchical model: we have found that performance on the same-different task declined when participants were given instructions to perform it by carrying out an egocentric perspective transformation (Zacks and Tversky, 2000). (Conversely, performance in the leftright task declined when participants were asked to perform it by carrying out an object-based transformation.) If the object-based transformation includes the egocentric perspective transformation as a subcomponent, then asking participants to imagine an egocentric perspective transformation as part of performing the task should be consistent with what they do naturally and so shouldn't affect performance. In sum, we bel ieve the available data currently favor the double-dissociation model, but the current observations suggest that the hierarchical model is also a viable possibility.

The present data contribute to an emerging picture of how components of spatial reasoning can be implemented by isolable neural subsystems. The multiple systems framework provides a means of framing this picture, of formulating models of the relationship between classes of spatial transformations, patterns of behavioral performance, and neurophysiology.

\section{ACKN OWLEDGMENTS}

This research was supported in part by the Mallinckrodt Institute of Radiology. The authors thank Erbil Akbudak and Mark McAvoy for their assistance, and Avi Snyder and J oel Myerson for helpful discussions.

\section{REFERENCES}

Aguirre, G. K., and D'Esposito, M. 1997. Environmental knowledge is subserved by separable dorsal/ventral neural areas. J . Neurosci. 17: 2512-2518.

Alivisatos, B., and Petrides, M. 1997. Functional activation of the human brain during mental rotation. Neuropsychologia 35: 111118.

Barnes, J ., Howard, R. J ., Senior, C., Brammer, M., Bullmore, E. T., Simmons, A., Woodruff, P., and David, A. S. 2000. Cortical activity during rotational and linear transformations. Neuropsychologia 38: $1148-1156$.

Bonda, E., Petrides, M., Ostry, D., and Evans, A. 1996. Specific involvement of human parietal systems and the amygdala in the perception of biological motion. J . Neurosci. 16: 3737-3744.

Boynton, G. M., Engel, S. A., Glover, G. H., and Heeger, D. J . 1996. Linear systems analysis of functional magnetic resonance imaging in human V1. J. Neurosci. 16: 4207-4221.

Buckner, R. L., and Braver, T. S. 1999. Event related functional MRI. In Functional MRI (P. Bandettini and C. Moonen, Eds.), pp. 441450. Springer-Verlag, Germany.

Burock, M. A., and Dale, A. M. 2000. Estimation and detection of event-related $\mathrm{fMRI}$ signals with temporally correlated noise: $A$ statistically efficient and unbiased approach. Hum. Brain Mapp. 11: $249-260$.

Casey, B. J ., Thomas, K. M., Welsh, T. F., Badgaiyan, R. D., Eccard, C. H., J ennings, J. R., and Crone, E. A. 2000. Dissociation of response conflict, attentional selection, and expectancy with functional magnetic resonance imaging. Proc. Natl. Acad. Sci. USA 97: 8728-8733.

Cohen, J. D., MacWhinney, B., Flatt, M., and Provost, J . 1993. PsyScope: An interactive graphic system for designing and controlling experiments in the psychology laboratory using Macintosh computers. Behav. Res. Methods Instruments Comput. 25: 257271.

Cohen, M. S., Kosslyn, S. M., Breiter, H. C., Digirolamo, G. J ., Thompson, W. L., Anderson, A. K., Bookheimer, S. Y., Rosen, B. R., and Belliveau, J. W. 1996. Changes in cortical activity during mental rotation: A mapping study using functional MRI. Brain 119: $89-100$.

Conturo, T. E., McKinstry, R. C., Akbudak, E., Snyder, A. Z., Yang, T. Z., and Raichle, M. E. 1996. Sensitivity optimization and experimental design in functional magnetic resonance imaging. Soc. Neurosci. Abstracts 22: 7.

Corballis, M. C. 1997. Mental rotation and the right hemisphere. Brain Lang. 57: 100-121.

Corbetta, M., Akbudak, E., Conturo, T. E., Snyder, A. Z., Ollinger, J. M., Drury, H. A., Linenweber, M. R., Petersen, S. E., Raichle, M. E., Van Essen, D. C., and Shulman, G. L. 1998. A common network of functional areas for attention and eye movements. Neuron 21: 761-773.

De Renzi, E. 1982. Disorders of Space Exploration and Cognition. Wiley, Chichester.

De Yoe, E. A., Bandettini, P., Neitz, J., Miller, D., and Winans, P. 1994. Functional magnetic resonance imaging (fMRI) of the human brain. J . Neurosci. Methods 54: 171-187.

DeSoto, M. C., Fabiani, M., Geary, D. C., and Gratton, G. 2001. When in doubt, do it both ways: Brain evidence of the simultaneous activation of conflicting motor responses in a spatial stroop task. J . Cogn. Neurosci. 13: 523-536.

Ditunno, P. L., and Mann, V. A. 1990. Right hemisphere specialization for mental rotation in normals and brain damaged subjects. Cortex 26: 177-188.

Farrell, M. J . 1996. Topographical disorientation. Neurocase 2: 509520. 
Funnell, M. G., J ohnson, S. H., and Gazzaniga, M. S. 2001. Hemispheric differences in egocentric and allocentric mental rotation: Evidence from $\mathrm{fMRI}$ and a split-brain patient. Paper presented at the Annual Meeting of the Cognitive Neuroscience Society, New York.

Gandhi, S. P., Heeger, D. J ., and Boynton, G. M. 1999. Spatial attention affects brain activity in human primary visual cortex. Proc. Natl. Acad. Sci. USA 96: 3314-3319.

Grossman, E., Donnelly, M., Price, R., Pickens, D., Morgan, V., Neighbor, G., and Blake, R. 2000. Brain areas involved in perception of biological motion. J . Cogn. Neurosci. 12: 711-720.

Gusnard, D. A., and Raichle, M. E. 2001. Searching for a baseline: Functional imaging and the resting human brain. Nature Rev. Neurosci. 2: 685- 694.

Harris, I. M., Egan, G. F., Sonkkila, C., Tochon-Danguy, H. J ., Paxinos, G., and Watson, J. D. G. 2000. Selective right parietal lobe activation during mental rotation: A parametric PET study. Brain 123: 65-73.

Huk, A. C., and Heeger, D. J . 2000. Task-related modulation of visual cortex. J . Neurophysiol. 83: 3525-3536.

Huttenlocher, J ., and Presson, C. C. 1973. Mental rotation and the perspective problem. Cogn. Psychol. 4: 277-299.

J olicoeur, P. 1985. The time to name disoriented natural objects. Memory Cogn. 13: 289-303.

J ust, M. A., and Carpenter, P. A. 1985. Cognitive coordinate systems: Accounts of mental rotation and individual differences in spatial ability. Psychol. Rev. 92: 137-172.

Kosslyn, S. M. 1994. Imageand Brain: The Resolution of thel magery Debate MIT Press, Cambridge, MA.

Kosslyn, S. M., DiGirolamo, G. J ., Thompson, W. L., and Alpert, N. M. 1998. Mental rotation of objects versus hands: Neural mechanisms revealed by positron emission tomography. Psychophysiology 35: 151-161.

Kwong, K. K., Belliveau, J. W., Chesler, D. A., Goldberg, I. E., Weisskoff, R. M., Poncelet, B. P., Kennedy, D. N., and Hoppel, B. E. 1992. Dynamic magnetic resonance imaging of human brain activity during primary sensory stimulation. Proc. Natl. Acad. Sci. USA 89: 5675-5679.

Lancaster, J . L., Woldorff, M. G., Parsons, L. M., Liotti, M., Freitas, C. S., Rainey, L., Kochunov, P. V., Nickerson, D., Mikiten, S. A., and Fox, P. T. 2000. Automated Talairach atlas labels for functional brain mapping. Hum. Brain Mapp. 10: 120-131.

Logothetis, N. K., Pauls, J., Augath, M., Trinath, T., and Oeltermann, A. 2001. Neurophysiological investigation of the basis of the fMRI signal. Nature 412: 150-157.

McAvoy, M., and Ollinger, J. M. 2001. Cluster size thresholds for assessment of significant activation in fMRI. Paper presented at the Human Brain Mapping, J une 10-14. Brighton, UK.

Miezin, F. M., Maccotta, L., Ollinger, J. M., Petersen, S. E., and Buckner, R. L. 2000. Characterizing the hemodynamic response: Effects of presentation rate, sampling procedure, and the possibility of ordering brain activity based on relative timing. Neurol mage 11: 735-759.

Ogawa, S., Lee, T. M., Kay, A. R., and Tank, D. W. 1990. Brain magnetic resonance imaging with contrast dependent on blood oxygenation. Proc. Natl. Acad. Sci. USA 87: 9868-9872.

Ojemann, J. G., Akbudak, E., Snyder, A. Z., McKinstry, R. C., Raichle, M. E., and Conturo, T. E. 1997. Anatomic localization and quantitative analysis of gradient refocused echo-planar fMRI susceptibility artifacts. Neurol mage 6: 156-167.

Ollinger, J . M., Corbetta, M., and Shulman, G. L. 2001. Separating processes within a trial in event-related functional MRI II: Analysis. Neurol mage 13: 218-229.
Ollinger, J . M., and McAvoy, M. 2000. A homogeneity correction for post-hoc ANOVAs in fMRI. Paper presented at the 6th Annual Meeting of the Organization for Human Brain Mapping, San Antonio, TX.

Ollinger, J . M., Shulman, G. L., and Corbetta, M. 2001. Separating processes within a trial in event-related functional MRI I: The method. Neurol mage 13: 210-217.

Parsons, L. M. 1987. Imagined spatial transformation of one's body. J . Exp. Psychol. Gen. 116: 172-191.

Parsons, L. M., Fox, P. T., Downs, J. H., Glass, T., Hirsch, T. B., Martin, C. C., J erabek, P. A., and Lancaster, J . L. 1995. Use of implicit motor imagery for visual shape discrimination as revealed by PET. Nature (London) 375: 54-58.

Pegna, A. J ., Khateb, A., Spinelli, L., Seeck, M., Landis, T., and Michel, C. M. 1997. Unraveling the cerebral dynamics of mental imagery. Hum. Brain Mapp. 5: 410- 421.

Pierret, A., Peronnet, F., and Thevenet, M. 1994. An electrophysiological study of the mental rotation of polygons. Neuroreport 5: 1153- 1156.

Podgorny, P., and Shepard, R. N. 1978. Functional representations common to visual perception and imagination. J . Exp. Psychol. Hum. Percept. Perform. 4: 21-35.

Poldrack, R. A., and Gabrieli, J . D. 2001. Characterizing the neural mechanisms of skill learning and repetition priming: Evidence from mirror reading. Brain 124: 67- 82.

Presson, C. C. 1982. Strategies in spatial reasoning. J . Exp. Psychol. Learn. Memory Cogn. 8: 243-251.

Pylyshyn, Z. W. 1979. The rate of mental rotation of images: A test of a holistic analogue hypothesis. Memory Cogn. 7: 19-28.

Raichle, M. E., MacLeod, A. M., Snyder, A. Z., Powers, W. J ., Gusnard, D. A., and Shulman, G. L. 2001. A default mode of brain function. Proc. Natl. Acad. Sci. USA 98: 676-682.

Rao, S. M., Binder, J. R., Bandettini, P. A., and Hammeke, T. A. 1993. Functional magnetic resonance imaging of complex human movements. Neurology 43: 2311-2318.

Ratcliff, G. 1979. Spatial thought, mental rotation and the right cerebral hemisphere. Neuropsychologia 17: 49-54.

Richter, W., Somorjai, R., Summers, R., J armasz, M., Menon, R. S., Gati, J. S., Georgopoulos, A. P., Tegeler, C., Ugurbil, K., and Kim, S. G. 2000. Motor area activity during mental rotation studied by time-resolved single-trial fMRI. J . Cogn. Neurosci. 12: 310-320.

Richter, W., Ugurbil, K., Georgopoulos, A., and Kim, S. G. 1997. Time-resolved fMRI of mental rotation. Neuroreport 8: 3697-3702.

Rosler, F., Heil, M., Bajric, J., and Pauls, A. C. 1995. Patterns of cerebral activation while mental images are rotated and changed in size. Psychophysiology 32: 135-149.

Rypma, B., Debell, M. A., Gabrieli, J . D. E., Prabakharan, V., Zabinski, M. F., Desmond, J. E., and Glover, G. H. 1996. Functional MRI studies of mental rotation and object identification processes. Soc. Neurosci. Abstracts 22: 720.

Semmes, J., Weinstein, S., Ghent, L., and Teuber, H.-L. 1963. Correlates of impaired orientation in personal and extrapersonal space. Brain 86: 747-772.

Shepard, R. N. 1994. Perceptual-cognitive universals as reflections of the world. Psychonom. Bull. Rev. 1: 2-28.

Shepard, R. N., and Cooper, L. A. 1982. Mental Images and Their Transformations. MIT Press, Cambridge, MA.

Shepard, R. N., and Metzler, J. 1971. Mental rotation of threedimensional objects. Science 171: 701-703.

Shulman, G. L., Corbetta, M., Buckner, R. L., Fiez, J . A., Miezin, F. M., Raichle, M. E., and Petersen, S. E. 1997. Common blood flow changes across visual tasks: I. I ncreases in subcortical structures and cerebellum but not in nonvisual cortex. J . Cogn. Neurosci. 9: 624- 647. 
Shulman, G. L., Fiez, J . A., Corbetta, M., and Buckner, R. L. 1997. Common blood flow changes across visual tasks: II. Decreases in cerebral cortex. J . Cogn. Neurosci. 9: 648-663.

Somers, D. C., Dale, A. M., Seiffert, A. E., and Tootell, R. B. 1999. Functional MRI reveals spatially specific attentional modulation in human primary visual cortex. Proc. Natl. Acad. Sci. USA 96: 16631668.

Tagaris, G. A., Kim, S.-G., Strupp, J . P., Andersen, P., U gurbil, K., and Georgopoulos, A. P. 1997. Mental rotation studied by functional magnetic resonance imaging at high field (4 Tesla): Performance and cortical activation. J . Cogn. Neurosci. 9: 419432.

Talairach, J ., and Tournoux, P. 1988. Co-planar Stereotaxic Atlas of the Human Brain: 3-Dimensional Proportional System, an Approach to Cerebral I maging. Thieme, Stuttgar.

Vingerhoets, G., Santens, P., Van Laere, K., Lahorte, P., Dierckx, R. A., and De Reuck, J. 2001. Regional brain activity during different paradigms of mental rotation in healthy volunteers: $A$ positron emission tomography study. Neuroimage 13: 381-391.

Warrington, E. K., J ames, M., and Maciejewski, C. 1986. The WAIS as a lateralizing and localizing diagnostic instrument: A study of 656 patients with unilateral cerebral lesions. Neuropsychologia 24: 223-239.
Wijers, A. A., Otten, L. J ., Feenstra, S., and Mulder, G. 1989. Brain potentials during selective attention, memory search, and mental rotation. Psychophysiology 26: 452- 467.

Wraga, M., Creem, S. H., and Proffitt, D. R. 1999. The influence of spatial reference frames on imagined object- and viewer rotations. Acta Psychol. (Amsterdam) 102: 247-264.

Wraga, M., Creem, S. H., and Proffitt, D. R. 2000. Updating scenes after object- and viewer-rotations. J . Exp. Psychol. Learn. Memory Cogn. 26: 151-168.

Yoshino, A., Inoue, M., and Suzuki, A. 2000. A topographic electrophysiologic study of mental rotation. Cogn. Brain Res. 9: 121-124.

Zacks, J ., Rypma, B., Gabrieli, J ., Tversky, B., and Glover, G. 1999. I magined transformations of bodies: An fMRI study. Neuropsychologia 37: 1029-1040.

Zacks, J . M., Braver, T. S., Sheridan, M. A., Donaldson, D. I., Snyder, A. Z., Ollinger, J. M., Buckner, R. L., and Raichle, M. E. 2001. Human brain activity time-locked to perceptual event boundaries. Nature Neurosci. 4: 651- 655.

Zacks, J . M., Mires, J ., Tversky, B., and Hazeltine, E. Mental spatial transformations of objects and perspective. Spatial Cogn. Comput., in press.

Zacks, J. M., and Tversky, B. 2000. Mental spatial transformations of bodies and objects. Abstracts Psychonom. Soc. 4: 72. 